\title{
Francisco González Medrano
}

\section{9-2017}

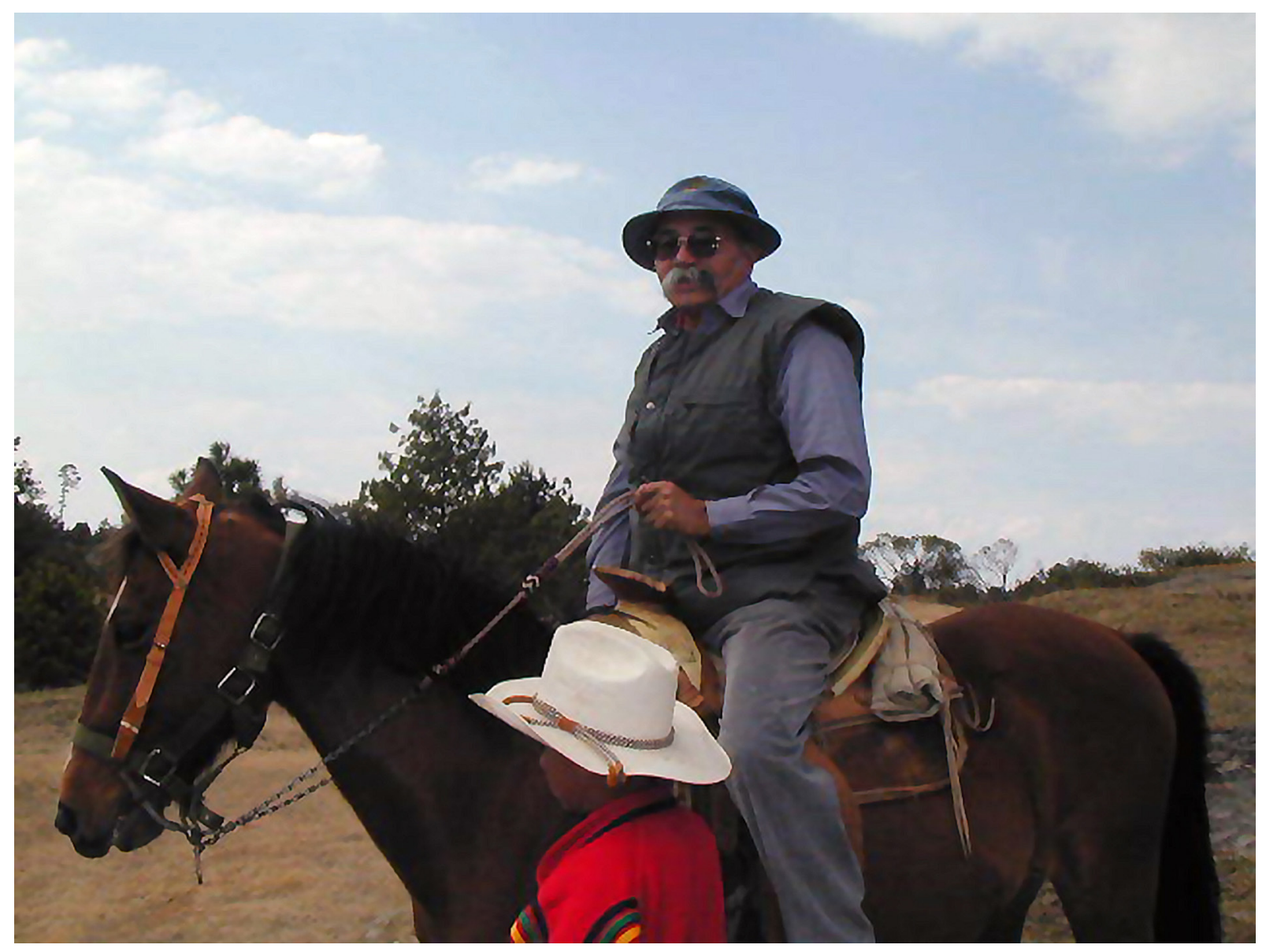

Francisco González Medrano (Fototeca del IB-UNAM)

\section{Luis Hernández Sandoval' [D}

Fue conocido de diversas formas, como el Maestro González Medrano, como Pancho González Medrano, Pancho Medrano o simplemente Pancho. En adelante me voy a referir a él sólo como El Maestro o el Maestro Medrano, pues así le decía cuando fui su alumno.

El Maestro nació en la Ciudad de México el 13 de enero de 1939 y estudió Biología en la Facultad de Ciencias de la Universidad Nacional Autónoma de México de 1960 a 1966, titulándose con la tesis "La vegetación del noreste de Tamaulipas". Fueron épocas difíciles para él, pues para sostenerse tuvo literalmente que pelear profesionalmente para salir adelante, hasta que el Maestro Javier Valdés lo invitó a trabajar como técnico del Jardín Botánico del Instituto de Biología de la UNAM.

A partir de ese momento, toda su vida académica la dedicó al conocimiento y estudio de la vegetación y la flora de México, principalmente de las zonas áridas y semiáridas. Quizá después de su tesis, el parteaguas que lo

1 Universidad Autónoma de Querétaro, Facultad de Ciencias Naturales, Biología. luishs@uaq.mx DOl: http://dx.doi.org/10.21829/abm119.2017.1227

Citar como: Hernández Sandoval, L. 2017. Obituario Francisco González Medrano. Acta Botanica Mexicana 119: 15-20. DOI: http://dx.doi.org/10.21829/ abm119.2017.1227 
llevó a esto fue la invitación del Dr. Arturo Gómez Pompa a trabajar en un estudio, visionario para su época, sobre el impacto sobre los ecosistemas y la zona agrícola por la construcción de la presa Las Adjuntas (Vicente Guerrero) en Tamaulipas. Desafortunadamente, las propuestas del informe final en 1971, según el Dr. Gómez Pompa y el Maestro, nunca fueron tomadas en cuenta. Sin embargo, además de que obtuvieron una mención honorífica por este trabajo en un premio que organizaba el Banco Nacional de México, el trabajo fue la base para muchos otros estudios de impacto ambiental en México y, sobre todo, para esa relación del Maestro Medrano con la vegetación de las zonas áridas y semiáridas. Por su dedicación al estudio sobre estos ecosistemas, pudo publicar diversos artículos, capítulos de libros, libros y documentos en los que describió la vegetación, nuevas especies para la ciencia, dos géneros nuevos aún válidos, uno de Celastraceae (Rzedowskia) y otro de Lamiaceae (Neoeplingia), así como usos de plantas y estrategias de manejo y conservación. Su trabajo de 2003 sobre "Las Comunidades Vegetales de México" presenta una síntesis de todo lo que sabía de su experiencia en campo y de sus ca. 22,000 ejemplares colectados, en el que hace una propuesta integral con lo mejor de las diversas clasificaciones de tipos de vegetación del país.

Debido a su amplia experiencia, le valió ser asesor de diferentes instituciones académicas y de gobierno en el país, tales como la Comisión Nacional para las Zonas Áridas (CONAZA), la Comisión Técnico Consultiva para los Coeficientes de Agostadero (COTECOCA), la Secretaría de Desarrollo Urbano y Ecología (SEDUE), el Consejo Nacional de Ciencia y Tecnología (CONACYT), la Comisión Nacional para el Conocimiento, Uso y Manejo de la Biodiversidad (CONABIO), así como la Secretaría de Medio Ambiente, Recursos Naturales y Pesca (SEMARNAP) y ahora Secretaría de Medio Ambiente y Recursos Naturales (SEMARNAT). Igualmente, desarrolló otros estudios o colaboraciones relevantes financiados por estas instituciones, tales como proyectos en la cuenca del Río Cutzamala, el Inventario Nacional Forestal o propuestas de conservación de las Islas Marías y las Revillagigedo.
Fue miembro activo de la Sociedad Botánica de México y en el período 1978-1979 fungió como su presidente, época en la que se promovió el conocimiento de la flora y vegetación de México a través de salidas de campo, principalmente los fines de semana. Como reconocimientos, la Universidad Autónoma de Tamaulipas designó al Jardín Botánico de su Centro Universitario con su nombre y tanto la Universidad Autónoma de Sonora como el Instituto de Biología de la UNAM le otorgaron reconocimientos por su labor académica. Además, algunas especies botánicas llevan su nombre, como Bursera medranoana Rzedowski \& Ortíz. En la publicación original (Rzedowski \& Ortíz, 1988) se asienta que "el nombre de la especie se dedica como homenaje al M. en C. Francisco González Medrano, prestigiado botánico mexicano y activo promotor de los estudios de la flora y vegetación de las barrancas semiáridas del estado de Hidalgo. El maestro Medrano herborizó los primeros ejemplares de este taxón".

Sin embargo, el otro aspecto que hizo al Maestro Medrano un profesionista excepcional fue su capacidad de enseñanza y de formar botánicos y ecólogos.

Impartió cursos, seminarios y talleres a nivel licenciatura, posgrado o de divulgación en muchas instituciones, pero principalmente en la Universidad Nacional Autónoma de México, en la Universidad Autónoma Metropolitana unidades Iztapalapa y Xochimilco, en la Universidad Autónoma de Morelos y en la Universidad Autónoma de Tamaulipas. Además, ocupó diversas cátedras, como Profesor Titular por oposición en la Cátedra de Botánica IV en la Licenciatura y en la División de Estudios de Posgrado en Ciencias Biológicas de la UNAM, sobre Biología Ambiental (Ecosistemas Naturales y Restauración Ambiental), la Cátedra Divisional "Dr. Enrique Beltrán Castillo" en la División de Ciencias Biológicas y de la Salud en la UAM-Xochimilco y como Profesor Extraordinario en la Universidad Autónoma del Estado de Morelos. En todas estas instituciones dirigió más de 40 tesis, tres de premiadas y más de 40 Seminarios de Investigación en el Posgrado de la Facultad de Ciencias de la UNAM y en la Licenciatura en Biología en la UAM-Iztapalapa. Una larga lista de investigadores se formó con el Maestro, ya 


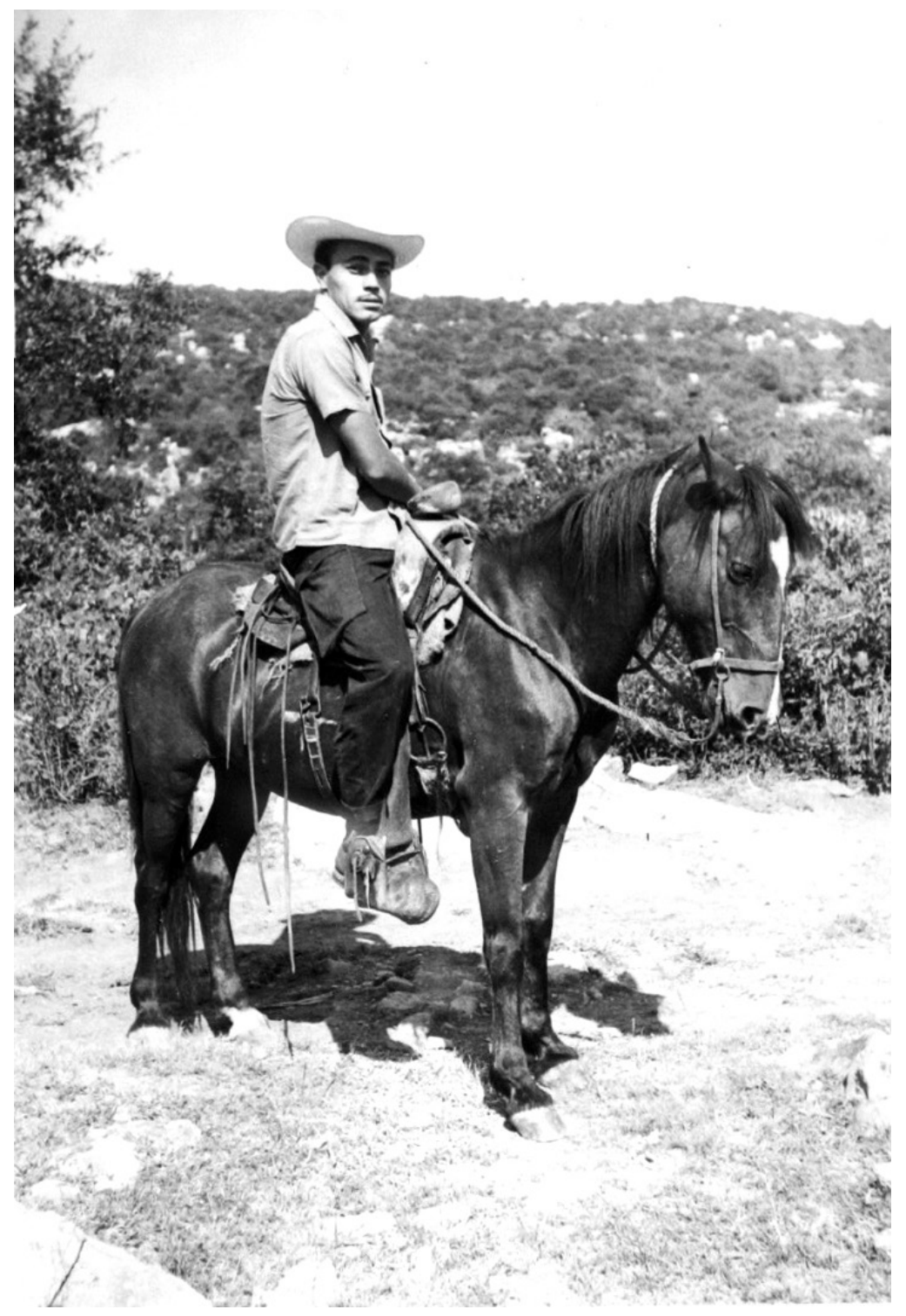

Francisco González Medrano (Fototeca del IB-UNAM)

fuera como tesistas o aprendiendo de plantas en sus salidas de campo. Solo por citar a algunos: Fernando Chiang Cabrera, Clara Hilda Ramos Álvarez, Rodolfo Dirzo Mijares, Enrique Martínez y Ojeda, María Luisa Piñol Gómez, Gustavo Soria Rocha, Aurora Chimal Hernández, Alejandro Castellanos Villegas, Guadalupe Ramos Espinoza, Patricia Hiriart Valencia, Gilda Ortíz Calderón, Francisco Espinoza García, Patricia Dávila Aranda, José Luis Villaseñor Ríos, Víctor Jaramillo Luque, Rosalinda Medina Lemos, Esteban Martínez Salas, Alfonso Valiente Banuet, Mahinda Martínez y Díaz, Alejandro Casas Fernández,
Juan Torres Guevara, Verónica Juárez Jaimes, José Antonio Reyes-Agüero, Arturo Mora Olivo y José Guadalupe Martínez Ávalos, entre muchos otros.

Con el Maestro aprendías a distinguir los tipos de vegetación de una forma fácil y rápida y además te enseñaba los nombres de las plantas de manera, digamos "sui generis". Tenías que olerlas, tocarlas, estrujarlas, descarapelar la corteza y en muchos casos probarlas. De las más interesantes y una que nunca olvidabas era la del experimento genético, en donde, según el Maestro era, "como cuando la gente puede enrollar la lengua y otros no" y nos 
daba a probar la planta en cuestión diciendo que algunos sentirían sabor amargo y otros no. Esta no era otra que Castela (Castelaria) tortuosa y obviamente todos sentíamos ese amargor terrible del "chaparro amargoso", especie y nombre común que después de eso no olvidas jamás. Otra enseñanza inolvidable era cuando te mandaba a colectar ejemplares de Cecropia obtusifolia y ya que regresabas todo maltratado por las hormigas te decía "eso que lo picó se llama Atta mexicana". Lo peor era si estabas en un chaparral y el Maestro encontraba "piñones del desierto" pero esa historia ya la sabe todo el mundo.

Para nosotros, el Maestro se sabía todas las plantas del campo y recuerdo que una vez le pregunté que qué hacía si alguien le preguntaba alguna de la que no supiera su nombre, entonces me dijo, "esa la tira compañero y así como sin querer, la pisa y dice ¡ay! a ver si la vemos al rato y se va por otro lado para que no la vuelvan a encontrar". Por supuesto que era broma, pues si no las sabía a la próxima nos decía "qué creen, ya supe que era y nos decía el nombre de la especie". El caso es que además de aprender vegetación y flora de esa manera, te divertías a morir por su forma de enseñar, porque organizaba carreras a ver quién subía más rápido un cerro; porque en las noches hacíamos sesiones interminables de chistes al calor de una fogata y de un tequila; porque aprendías albures, como el género tipo de la familia Melastomataceae; a esquivar "mala mujer" (Cnidoscolus multilobus) o "huizaches" (Acacia spp.) cuando nos mandaba al techo de la camioneta para ver mejor el camino o si llevabas la ventana abierta y el brazo afuera, entonces desviaba la camioneta sobre ellas; aprendías a conocer México y miles caminos ¡los cuales recordaba todos!; a arreglar camionetas siempre en riesgo de que nos dejaran en la sierra. No podré olvidar la travesía de cruzar a caballo durante varios días parte de la Sierra Madre Oriental a través del Cañon del Chihue y del Río Guayalejo, desde San Antonio, Jaumave hasta Llera, Tamaulipas, colectando con el Maestro, con Ricardo Moldzaski y Manuel Yáñez muchísimos números de plantas. Y por supuesto, dormir en la camioneta en el día era peligroso, so pena de brincar hasta el techo con el grito de ¡coyoooote! (el cual puede haber surgido en cualquier momento debido a su inventiva, pero una vez me contó que su origen venía de que escuchó al Ing. Efraím Hernández Xolocotzi, decir que "los estudiantes de las instituciones públicas podían aprender con muy poco dinero y sus maestros podían darles clases y tener un sueldo, gracias a los pocos mexicanos que pagan sus impuestos y a los pocos políticos que lo distribuyen bien, entonces todos teníamos que aprovechar el tiempo para aprender al máximo pues nos debíamos en gran parte a esas personas”). ¿Cómo no valorar todas estas enseñanzas?

Descanse en paz Maestro Francisco González Medrano, lo despedimos con un agradecimiento infinito, diciendo una de sus frases favoritas...

“así es esto de las zonas áridas".

\section{AgRAdECIMIENTOS}

A Fernando Chiang por proveer información y a Mahinda Martínez por traer varios recuerdos del Maestro y por revisar el escrito.

\section{LITERATURA REVISADA}

Butanda, A. y G. Calderón de Rzedowski. 2001. Los personajes y los hechos históricos en las contribuciones taxonómicas de Jerzy Rzedowski. En: Jerzy Rzedowski Rotter, Doctor Honoris Causa de la Universidad Michoacana de San Nicolás de Hidalgo. Instituto de Investigaciones Históricas, Universidad Michoacana de San Nicolás de Hidalgo. Morelia, México.

Gómez-Pompa, A. 2016. Mi vida en las selvas tropicales. Memorias de un botánico. http://www.reservaeleden.org/ agp/pdf/AutobiografiaAGP.pdf (consultado enero 2017)

Herrera, T. y A. Butanda. 2009. La Botánica en México. EM$\mathrm{C}=$ COSMOS. Botánica. Universidad Autónoma Metropolitana-Iztapalapa. http://www.izt.uam.mx/cosmosecm/ BOTANICA.html (consultado enero 2017).

Rzedowski, J. y E. Ortíz. 1988. Estudios quimiotaxonómicos de Bursera (Burseraceae). II. Una especie nueva de origen híbrido de la barranca de Tolantongo, estado de Hidalgo. Acta Botanica Mexicana 1: 11-19. DOI: http://dx.doi. org/10.21829/abm1.1988.559 
Como anexo se presenta una serie de publicaciones y documentos relevantes del Maestro:

González M., F. 1971. Vegetación. En: A. Gómez-Pompa, Estudio Ecológico de la zona de Las Adjuntas, Tamaulipas. Informe Final. Responsable, Universidad Nacional Autónoma de México-Secretaría de Agricultura y Recursos Hidráulicos. México, D.F., México. Pp. 974.

González-Medrano, F. 1972. Una nueva Frankeniaceae del norte de México. Boletín de la Sociedad Botánica de México 32: sin pág.

González-Medrano, F. 1972. La vegetación del noreste de Tamaulipas. Anales del Instituto de Biología, Universidad Nacional Autónoma de México Serie Botánica 43: 11-50.

Ramos, C. H. y F. González Medrano. 1972. La Vegetación de la zona árida veracruzana. Anales del Instituto de Biología, Universidad Nacional Autónoma de México, Serie Botánica 43: 77-79.

González M., F. 1972. Excursión al centro y noreste. En: Guías botánicas de excursiones en México. Sociedad Botánica de México. México, D.F., México. pp. 225-228.

González M., F. y H. Sánchez Mejorada. 1972. Excursión a la Barranca de Meztitlán. En: Guías botánicas de excursiones en México. Sociedad Botánica de México. México, D.F., México. pp. 63-68.

González M., F. 1973. Estudio ecológico en la cuenca del Río Cutzamala, Estado de México, Guerrero y Michoacán. Informe Final. Universidad Nacional Autónoma de México-Secretaría de Recursos Hidráulicos.

Martínez y Ojeda, E. y F. González Medrano. 1977. Vegetación del sudeste de Tamaulipas, México. Biótica 2(2): 1-45.

Chiang, F. y F. González Medrano. 1979. Flora y Vegetación del Valle de Tehuacán (proyecto). Tesistas: J. L. Villaseñor, P. Dávila y V. Jaramillo.

Chiang, F. y F. González-Medrano. 1981. Nueva especie de Casimiroa (Rutaceae) de la zona árida oaxaqueño-poblana. Boletín de la Sociedad Botánica de México 41: 23-26.

González Medrano, F. 1981. Rzedowskia, un nuevo género de Celastraceae de México. Boletín de la Sociedad Botánica de México 41: 41-45.
Medrano, F. G. 1982. Mimosa martindelcampoi Medrano (Leguminosae), una especie nueva del sudoeste de Tamaulipas, México. Boletín de la Sociedad Botánica de México 43: 39-42.

Ramamoorthy, T. P., P. Hiriart-Valencia y F. González-Medrano. 1982. Neoeplingia Ramamoorthy, Hiriart \& Medrano (Labiatae) un nuevo género de Hidalgo, México. Boletín de la Sociedad Botánica de México 43: 61-66.

Hiriart, V. P. y F. González Medrano. 1983. Vegetación y Fitogeografía de la barranca de Tolantongo, Hidalgo, México. Anales del Instituto de Biología, Universidad Nacional Autónoma de México Serie Botánica ( $\mathrm{N}^{\circ}$ único): 29-96.

Jaramillo Luque, V. y F. González Medrano. 1983. Análisis de la vegetación arbórea en la Provincia Florística de Tehuacán-Cuicatlán. Boletín de la Sociedad Botánica de México 45: 49-64.

Medina-Lemos, R. y F. González Medrano. 1984. Una especie nueva de Stegnosperma (Phytolaccaceae) del suroeste de Puebla, México. Boletín de la Sociedad Botánica de México 46: 37-42.

Hernández, L. y F. González Medrano. 1984. La importancia de las plantas útiles en Tamaulipas. Tamaholipa 1(1): 29.

González-Medrano, F. y F. Chiang. 1988. Diversidad florística y fitogeográfica de las zonas áridas del centro y sur de México. Resumen de ponencias. Simposio sobre diversidad biológica de México. Oaxtepec, México. pp. 34-36.

Hernández, L., C. González y F. González Medrano. 1991. Plantas útiles de Tamaulipas, México. Anales del Instituto de Biología, Universidad Nacional Autónoma de México, Serie Botánica 62(1): 1-38.

González-Medrano, F. 1993. Obito. Howard Scott Gentry (1908-1993). Boletín de la Sociedad Botánica de México 53: $135-140$.

Valiente Banuet, A., F. González Medrano y D. Piñero Dalmau. 1995. La vegetación selvática de la región de Gómez Farías, Tamaulipas, México. Acta Botanica Mexicana 33: 1-36. DOI: http://dx.doi.org/10.21829/abm33.1995.751

Reyes-Agüero, J. A., González-Medrano F. y J. D. GarcíaPérez. 1996. Flora vascular de la Sierra Monte Grande, municipio de Charcas, San Luis Potosí, México. Boletín de la Sociedad Botánica de México 58: 31-42. 
González-Medrano F. 1996. Algunos aspectos de la evolución de la vegetación de México. Boletín de la Sociedad Botánica de México 58: 129-136.

González-Medrano, F. y G. Hernández Mejía. 1998. Las Dolinas de Tamaulipas. Ciencias 50: 56-58.

González-Medrano, F. 1998. Lista florística preliminar de Tamaulipas. Informe final. Sistema Nacional de Información sobre Biodiversidad-Comisión nacional para el conocimiento y uso de la biodiversidad Proyecto No. P092. México, D.F., México.

González-Medrano, F. 1999. Adaptación del Sistema Nacional de Clasificación de la Vegetación (NVCS; 1993) de EE.UU. usado en los "GAP Analysis". Para la clasificación de la vegetación de la faja fronteriza del Río Bravo en México. Inédito.

Palacio Prieto, J. L., G. Bocco, A. Velázquez, J. F. Mas, F. Takaki, A. Victoria, L. Luna Gonzáles, G. Gómez Rodríguez, J. López García, M. Palma, I. Trejo Vázquez, A. Peralta, J. Prado Molina, A. Rodríguez Aguilar, R. Mayorga Saucedo y F. González Medrano. 2000. La condición actual de los recursos forestales en México: resultados del Inventario Forestal Nacional 2000. Investigaciones Geográficas 43: 183-203.
Velázquez, A., J. F. Mas, R. Mayorga Saucedo, J. L. Palacio, G. Bocco, G. Gómez Rodríguez, L. Luna Gonzáles, I. Trejo, J. López García, M. Palma, A. Peralta, J. Prado Molina y F. González Medrano. 2001. El Inventario Forestal Nacional 2000. Ciencias 64: 13-19.

González Medrano, F. 2003. Las comunidades vegetales en México: propuesta para la unificación de la clasificación y nomenclatura de la vegetación de México. Secretaría de Medio Ambiente y Recursos Naturales: Instituto Nacional de Ecología. México, D.F., México. 81 pp.

González-Medrano, F., J. L. Villaseñor y R. Medina. 2004. A new species of Gochnatia (Asteraceae, Mutisieae) from the Desert scrubland of the State of Hidalgo, Mexico. Novon 14(4): 434-436.

Cram, S. I. Sommer, L. M. Morales, O. Oropeza, E. Carmona y F. González-Medrano. 2006. Suitability of the vegetation types in Mexico's Tamaulipas state for the siting of hazardous waste treatment plants. Journal of Environmental Management 80(1): 13-24.

González-Medrano, F. 2012. Las zonas áridas y semiáridas de México y su vegetación. Instituto Nacional de Ecología, Secretaría de Medio Ambiente y Recursos Naturales. México, D.F., México. 173 pp. 